\title{
Role of a Nurse in Preventing the Diabetic Foot Storm
}

\author{
Mandira Gope $^{1}$, Ashok Dhanwal ${ }^{2}$ \\ ${ }^{1}$ Assistant Professor, Rajarajeswari College of Nursing, Bangalore. \\ ${ }^{2}$ Professor, M G College of Nursing, Delhi. \\ Corresponding Author: Mandira Gope
}

\begin{abstract}
Diabetes mellitus is a common epidemic and metabolic condition worldwide, which needs serious concern to avoid major complication. Out of several complications diabetic foot ulcer is the impenetrable one. 'Amit Jain' from India has classified 6 categories of diabetic foot problems and describes the pathway towards the diabetic foot storm (Apoorva H C \& Jain A K C, 2020). These categories and pathway is beneficial to construct preventive plan of action to detain diabetic foot storm. Diabetic foot storm encompasses different problem combined with diabetic foot condition. Nurses are playing vital role in every health care sector. They can save this storm with the knowledge of diabetic foot storm, pathway and different preventive strategies includes education, screening, awareness camping.
\end{abstract}

Key Words: Diabetic foot, Storm, Strategies, Prevention, Epidemic

\section{INTRODUCTION}

Diabetes mellitus is one of the most serious diseases in this century, which requires prevention and treatment. There are several known chronic complications like microvascular, macro vascular and neurological complications.

Among these complications of diabetes the most feared complications for diabetic patients are diabetic foot disease. The incidence of diabetic foot problem are increasing day by day resulting in hospitalized and increasing treatment cost of diabetic patient.

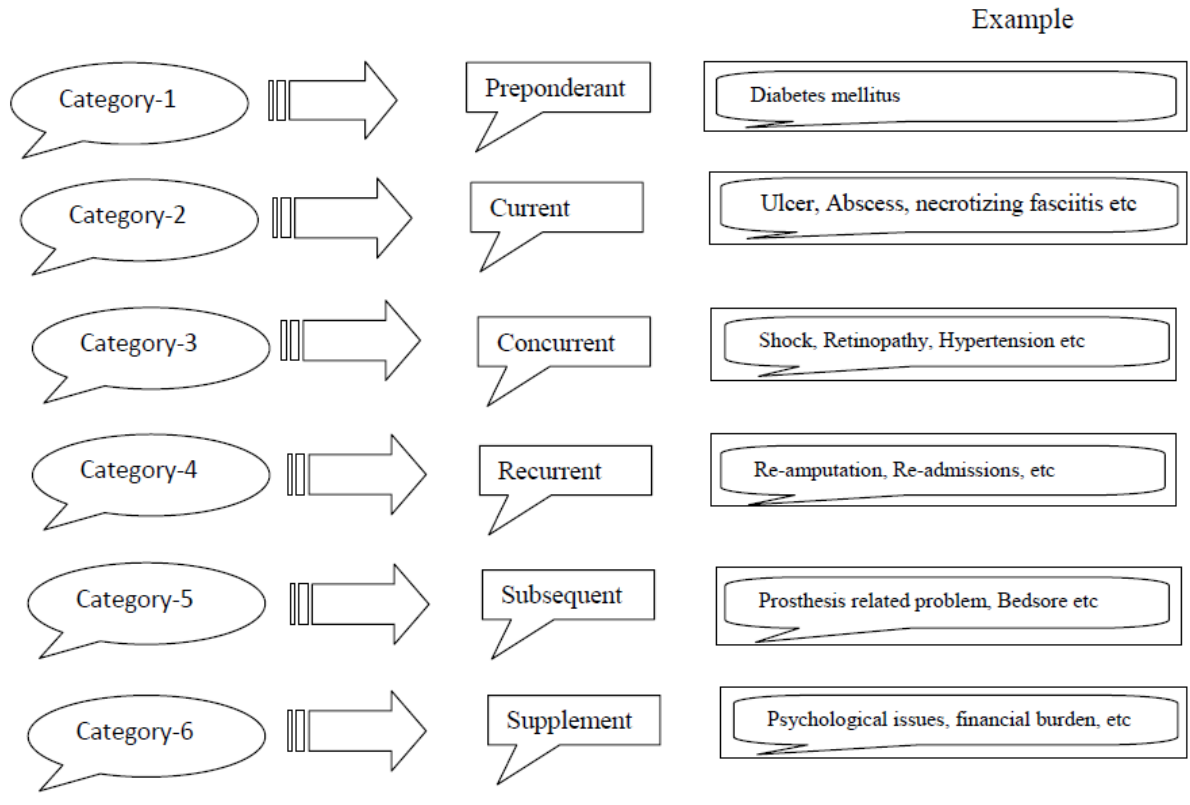

Figure: -1 Amit Jain's classification of problems of diabetic foot. 
It is noticed about $50 \%$ mortality rate is because of diabetic foot amputation. Recent studies reveal that drastic decrease in number of major amputation with the help of preventive education, screening and awareness of the condition.

The diabetic foot storm was recently contrived by Amit Jain from India, who designed triple assessment for diabetic foot in diabetes and futuristic system for diabetic foot surgery. The word 'diabetic foot storm' was extrapolated from the new classification of problems in diabetic foot.
According to Amit Jain's classification of problems of diabetic foot consist of 6 categories (Figure- 1) these are preponderant, current, concurrent, recurrent, subsequent, supplement.

According to this if patient has gone through 3 problems, then it is considering as a warning condition. If patient has gone through 4 problems, then it indicates impending storm. One it is reaching to 5 or more problems than it is consider as diabetic foot storm.

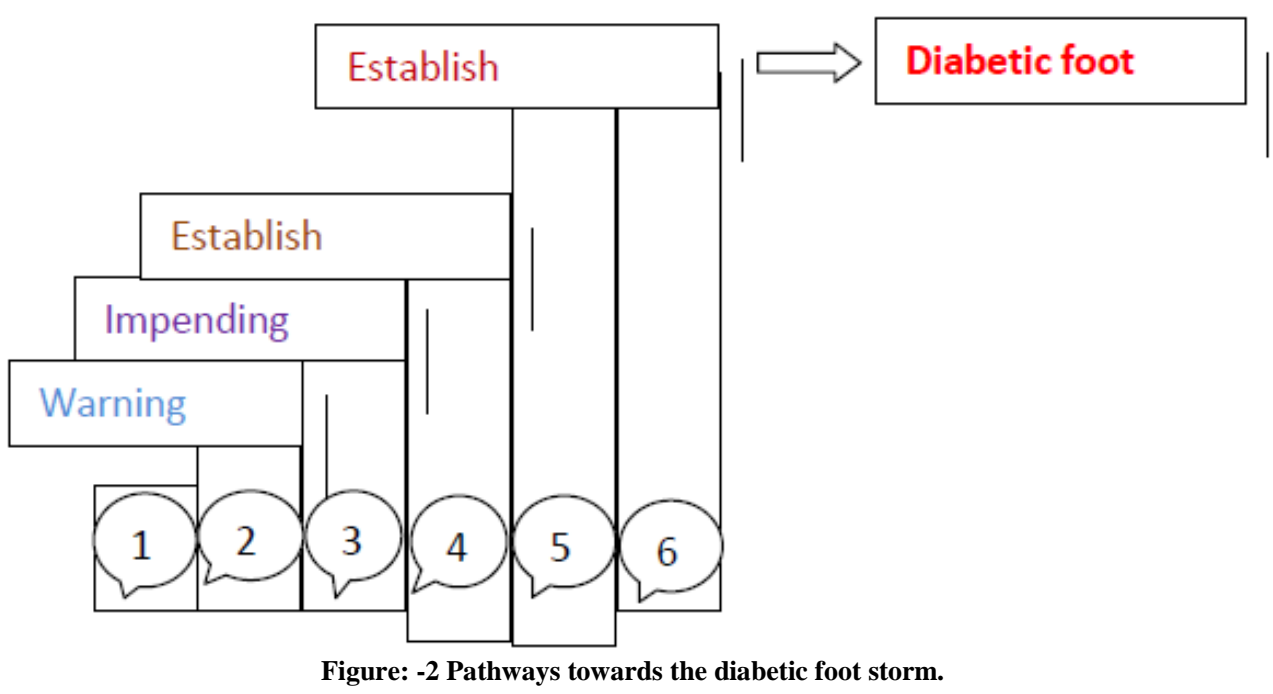

\section{Role of a nurse}

A nurse is taking integral part in patient care at hospital setting as well as community settings. Nurse should be aware of patient needs. Proper knowledge about the disease condition can help the nurse to understand the needs of the patient and to provide preventive care. It quite obvious that any of the diabetic setup like multidisciplinary clinic, hospital and institute would have general nurse assuming different role that has been instituted upon. In patient who is having diabetic foot often the nurses can be a part of both preventive strategies as well as therapeutic management like administration of drug, wound dressing, assist in investigation and assessment etc.

We know that diabetic foot storm is one of the most hazardous situations if it occurs to diabetic patient. Surprisingly most health care providers are not aware about diabetic foot storm. Nurses are the front line health worker and they should be aware of such consequences, their categories, and preventive measures that can be implemented.

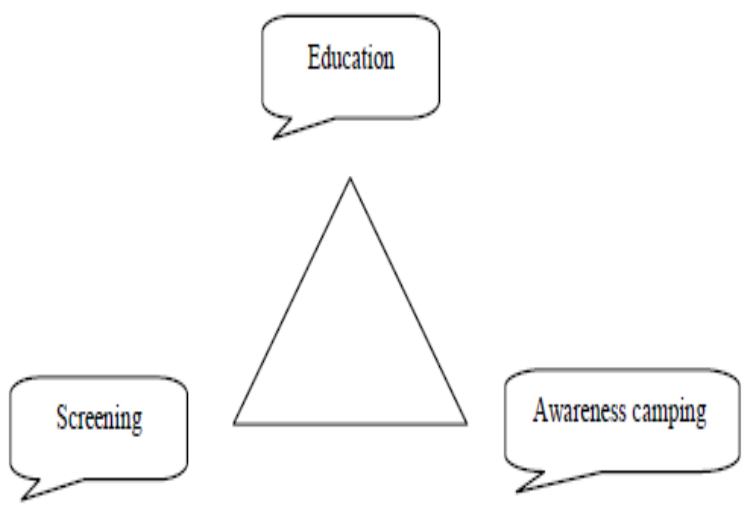

Figure: -3 Preventive Strategies for diabetic foot storm. 
Some of the strategies that has been adopted to prevent diabetic foot storm are education, screening and awareness (Figure3)

Nurses with knowledge of diabetic foot storm can educate the next group of nurses, general ward nurses and specialty clinic nurses. They can educate the patient and patient family about consequences diabetic foot storm. Proper education can help the patient in delay process of diabetic foot storm. If patient is aware about categories and developmental way of diabetic foot problem they will be conscious about their regular screening and follow-up which will delay development of diabetic foot storm and other associated problem. It will reduce ultimate economic burden and improve quality life span for diabetic patient. Education can prohibit the unusual diabetic complication, amputation, prosthesis related problem etc. Essential intervention in any disease is one of the most important preventive measures, like Amit Jain triple assessment. This simple screening tool consists of three component look, feel, and test. Being one of the simplest tool that requires minimum resources, minimum expertise and yet effectively addressed the triad of diabetic foot.

Since it does not required use of complicated gadgets and expensive training this screening tool can be used universally in health care settings. This screening strategies involves valuate of minimum parameters. The look component consists of examining 3 sites for any infection ulcer or pre-ulcer causing pathology, deformities and callosities. The look component involves seeing the dorsum, interdigital area and planter area that hardly take 3-4 sec.

The second component of the screening tool feel component. This component involves assessing whether the foot has adequate blood supply to rule out any underlying ischemia. Nurses are well trained throughout the curriculum in assessing the radial pulses was never implemented. Hence, the nurses can be trained or emphasis can be instituted to examine any of the distal foot pulses.

The test component involves assessing the underlying nerves to rule out neuropathy this screening tool gives a freedom to a health care professional to use any available method/instrument prevalent in their region that can be used in some of the known methods and instruments that can be use by nurses with minimum resource are tuning fork, monofilament, Ipsitouch test etc. If the centre is well equipped they can use expensive instruments like Biothesiometer for the screening at that centre. But for a large community setting a tuning fork, a monofilament is sufficient enough in the neuropathy screening. There are enough studies that shows that common bedside instrument and methods.

As a nursing educative what I observed is that the screening part has never been taught in the curriculum and also not implemented in the practice and since Amit Jain screening tool (Jain A K C, 2018) is one of the easiest tool with minimum resources and required skills, it can be implemented in nursing curriculum and practice which could bring a big impact in decreasing amputation worldwide. In view of Amit Jain screening tool being extremely easy both understand and performing extra burden in is not added to nursing care system. Recently the screening tool was given a scoring system and was well validate (Apoorva HC \& Jain A K C, 2020).

Awareness of diabetic foot problems the diabetic foot storm it's pathway etc to both heath care and non-health care profession is also one of the strategies in prevention displaying of the pathway of ulceration, amputation diabetic foot storm, in the lobbies, hospital campuses serves as good methods of bringing constant awareness of all. Community display or community awareness through play's can be done or even in nursing conferences, nursing workshop is another method of being awareness. 


\section{CONCLUSION}

It goes beyond doubt as a practicing nurse diabetic foot not being taught appropriately in curriculum. It's high time that the knowledge of diabetic foot storm and preventive strategies like education and screening becomes a day to day activity in a nurse's practice especially in centers there is diabetic patient. Nurses can be a good frontline team member in a diabetic foot team in preventive strategies. They can play an essential role from the time of the patient diagnosed as diabetic foot storm. The habit of screening should be instituted forum the undergraduate training it-self. So that it becomes a part of their practice in first stage. Amit Jain screening tool in the form of a triple assessment of foot as definitely easy, simple to perform requires minimum resources and expertise.

\section{Acknowledgement: None}

\section{Conflict of Interest: None}

\section{Source of Funding: None}

\section{REFERENCES}

1. Apoorva H C \& Jain A K C. (2020). Diabetic foot awareness - "From foot attack to diabetic foot storm". SAR Journal of Surgery, 1(3), 48-50.

2. Jain A K C. (2021). Supper modern diabetic foot surgery. Medicine Science International Medical Journal, 10(3).
3. Jain A K C. (2020). The diabetic foot storm. IAR Journal of Medical Science. 1(7), 321323.

4. Jain A K C, Apoorva HC \& Kishore K. (2019). Screening of diabetic foot through Amit Jain's triple assessment: A 10 to 20 second screening methods. International Journal of orthopedics Sciences. 5(2), 227229.

5. Apoorva H C \& Jain A K C. (2020). Strategies to prevent diabetic foot storm. International Journal of Surgery and Surgical Research, 2(1), 18-20.

6. Jain A, Kishore K, Harish K \& Suresh K. (2018). Amit Jain's triple assessment - a new screening method for the diabetic foot. Diabetic Foot Journal Middle East. 4(1).

\section{ABOUT AUTHORS}

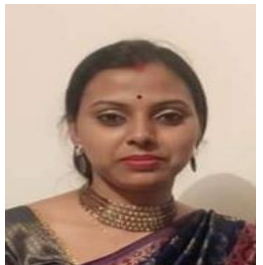

Primary Author: Mrs. Mandira Gope

Designation: Assistant

Professor, Rajarajeswari College of Nursing, Bangalore.

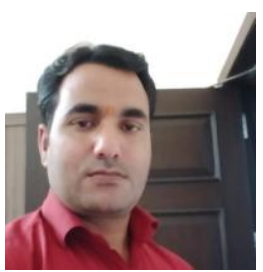

Secondary Author: Dr. Ashok Dhanwal Designation: Professor, M G College of Nursing, Delhi.

How to cite this article: Gope M, Dhanwal A. Role of a nurse in preventing the diabetic foot storm. International Journal of Research and Review. 2021; 8(8): 51-54. DOI: https://doi.org/ 10.52403/ijrr.20210809 